SACLAY-T05/012

\title{
Non-linear QCD evolution with improved triple-pomeron vertices
}

\author{
E. Iancu ${ }^{1}$ and D.N. Triantafyllopoulos \\ Service de Physique Théorique, CEA/DSM/SPhT, Unité de recherche associée au CNRS \\ (URA D2306), CE Saclay, F-91191 Gif-sur-Yvette, France
}

\begin{abstract}
In a previous publication, we have constructed a set of non-linear evolution equations for dipole scattering amplitudes in QCD at high energy, which extends the Balitsky-JIMWLK hierarchy by including the effects of fluctuations in the gluon number in the target wavefunction. In doing so, we have relied on the color dipole picture, valid in the limit where the number of colors is large, and we have made some further approximations on the relation between scattering amplitudes and dipole densities, which amount to neglecting the non-locality of the two-gluon exchanges. In this Letter, we relax the latter approximations, and thus restore the correct structure of the 'triple-pomeron vertex' which describes the splitting of one BFKL pomeron into two within the terms responsible for fluctuations. The ensuing triple-pomeron vertex coincides with the one previously derived by Braun and Vacca within perturbative QCD. The evolution equations can be recast in a Langevin form, but with a multivariable noise term with off-diagonal correlations. Our equations are shown to be equivalent with the modified version of the JIMWLK equation recently proposed by Mueller, Shoshi, and Wong.
\end{abstract}

$\overline{1}$ Membre du Centre National de la Recherche Scientifique (CNRS), France. 


\section{Introduction}

In a recent publication [1], we have shown that the Balitsky-JIMWLK (JalilianMarian-Iancu-McLerran-Weigert-Leonidov-Kovner) hierarchy of equations [2-5] previously proposed to describe the non-linear evolution of scattering amplitudes in QCD at high energy fails to include the effects of fluctuations in the gluon number in the target wavefunction, that were recently demonstrated to play an important role in the approach towards saturation and the unitarity limit [6,7]. Still in Ref. [1], we have then constructed a generalization of these equations which includes the relevant fluctuations in the limit where the number of colors is large $\left(N_{c} \gg 1\right)$ and in the kinematical region where these effects are expected to be important $[6,7]$, namely, in the low density regime at high transverse momenta (well above the target saturation momentum $[8,9]$ ). To that aim, we have relied on Mueller's color dipole picture [10-12], which is indeed known to provide a complete description of the target wavefunction (including the relevant fluctuations) in the approximation of interest. Besides, in order to simplify some of the technical manipulations in Ref. [1], we have performed some further approximations in the relation between scattering amplitudes and dipole densities, which are tantamount to neglecting the non-locality of the two-gluon exchanges in dipole-dipole scattering.

Although reasonable within the most interesting kinematical region — namely, within the intermediate regime at high momenta which is characterized by the BFKL 'anomalous dimension' $[8,13-17]$ - these additional approximations have ineluctably entailed a loss of accuracy, and also some conceptual shortcomings, for our final results in Ref. [1]. Specifically, the normalization of the fluctuation terms in our final equations there is afflicted with an unknown 'fudge' factor, and the non-locality structure of those terms is oversimplified. Because of that, these equations cannot be used, e.g., to study the transition towards the high $-Q^{2}$ evolution [18], which is expected to take place at sufficiently large transverse momenta. For instance, one would like to understand the interplay between the fluctuation-dominated dynamics predicted by the analysis in Refs. [1,7] (which should completely supersede the BFKL dynamics at very high energies) and the DGLAPlike dynamics [18] which should emerge at higher transverse momenta. But in order to do so, one would need the correct non-local structure of all the terms in the equations, including the fluctuation terms. Besides, the too drastic simplifications performed on the structure of the fluctuation terms leads to numerical difficulties which prevent a direct resolution of the hierarchy of equations proposed in Ref. [1] through iterations [19]. These difficulties are eliminated by an additional coarse-graining in impact parameter space, which leads to a mathematically well-defined Langevin equation [1] — the QCD analog of the stochastic Fisher-Kolmogorov-Petrovsky-Piscounov (sFKPP) equation [20]. But this final equation has the further drawback to ignore the impact-parameter dependence of the scattering amplitudes. Last but not least, we would like to be able to compare the newly proposed terms in Ref. [1] with related results in perturbative QCD [21-26]. As explained in [1], these terms describe pomeron splittings in the target wavefunction. The relevant triple-pomeron vertex has been computed in perturbative QCD in the recent years $[21,23,25,26]$, and we would like to identify this as a building block in our 
fluctuation terms.

For all these reasons it would be important to relax the additional approximations performed in Ref. [1], and thus obtain the complete equations valid to leading order at large $N_{c}$. This is the task that we shall undertake in this Letter. Specifically, by using the complete relations between scattering amplitudes and dipole densities valid in the dilute regime, we shall be able to restore the full non-locality of the fluctuation terms in the evolution equations for the amplitudes, and thus correct all the deficiencies alluded to before. In particular, in our new equations we shall recognize, as expected, the large $-N_{c}$ version of the triple-pomeron vertex describing pomeron splitting, in the form given by Braun and Vacca [23] (see also [22]). Since we knew already that the triple-pomeron vertex responsible for pomeron mergings is also included [25, 27], through the non-linear terms in our equations (which are the same as the corresponding terms in the BalitskyJIMWLK hierarchy [2-5]), we conclude that the new equations generate correctly, through iterations, the pomeron loops expected in perturbative QCD at large $N_{c}[11,12]$.

In view of numerical studies, it is often convenient to dispose of a stochastic formulation of a hierarchy of equations. We shall show that our new equations can be equivalently reformulated as a Langevin equation, which generalizes the one proposed in Ref. [1] by including the impact-parameter dependence and the full non-locality of the coefficient of the noise term. The counterpart of this is the fact that the new noise term depends upon three variables, and has non-diagonal correlations in two of them, which rises some doubts about his usefulness for practical calculations.

In this paper, we shall follow the same general strategy as in Ref. [1], namely, we shall view the high-energy evolution as the evolution of the target wavefunction, for which we shall use the dipole picture [10] in the dilute regime at high momenta and the JIMWLK evolution [3-5] in the high density regime near, or at, saturation. Very recently, Mueller, Shoshi, and Wong [28] have proposed a generalization of the JIMWLK equation which includes the effects of pomeron splitting in the dilute regime, at the same accuracy level as the dipole picture. We shall explicitly check that our new equations are indeed equivalent with the evolution described in Ref. [28]. In particular, the need for a non-diagonal noise term in order to achieve a stochastic formulation has been originally recognized in [28].

The paper is structured as follows: In Sect. 2, we shall construct the fluctuation term for the equation describing the evolution of the scattering amplitude for a pair of dipoles (which is the lowest equation in the hierarchy where this term enters). Then, in Sect. 3 , we shall generalize this construction to the equations of higher level in the hierarchy (pertinent to the scattering of $\kappa$ dipoles with $\kappa \geq 3$ ), and we shall introduce the alternative formulation of the hierarchy as a Langevin equation. In Sect. 4 we shall demonstrate the equivalence with the extended JIMWLK formalism of Ref. [28]. Finally, in Sect. 5 we shall analyze the structure of the triple-pomeron vertex encoded in our equation, and recognize this as the vertex originally computed by Braun and Vacca [23]. 


\section{The scattering amplitude for a pair of dipoles}

In this section we will derive the evolution equation for the amplitude describing the scattering of a dipole pair off an arbitrary hadronic target in the large $-N_{c}$ limit. We shall view the scattering in a frame in which most of the total rapidity $Y$, with $\bar{\alpha}_{s} Y>1$, is carried by the target (whose wavefunction is therefore highly evolved), whereas the projectile is simply a set of elementary dipoles. Also, we shall discuss explicitly only the contributions to the evolution arising from fluctuations in the gluon number in the target wavefunction. From the discussion in Refs. $[1,6,7]$, we expect such fluctuations to play an important role only in the tail of the distribution at large transverse momenta, where the gluon occupation factor is low (of order one), and the dipole picture is reliable (for large $N_{c}$ ). To study this low density region, we shall assume that the external dipoles composing the projectile have small transverse sizes (much smaller than the inverse of the target saturation momentum at their respective impact parameters).

Consider first the scattering of a single external dipole, with transverse coordinates $\boldsymbol{x}$ and $\boldsymbol{y}$ (for the quark and antiquark, respectively). In the present approximations, this dipole undergoes single scattering via two gluon exchange with any of the dipoles internal to the target. Thus, the corresponding scattering amplitude reads:

$$
\langle T(\boldsymbol{x}, \boldsymbol{y})\rangle_{Y}=\alpha_{s}^{2} \int \mathrm{d}^{2} \boldsymbol{u} \mathrm{d}^{2} \boldsymbol{v} \mathcal{A}_{0}(\boldsymbol{x}, \boldsymbol{y} \mid \boldsymbol{u}, \boldsymbol{v}) n_{Y}(\boldsymbol{u}, \boldsymbol{v})
$$

where, up to a factor $\alpha_{s}^{2}, \mathcal{A}_{0}$ is the amplitude for dipole-dipole scattering in the two-gluon exchange approximation and for large $N_{c}$ :

$$
\mathcal{A}_{0}(\boldsymbol{x}, \boldsymbol{y} \mid \boldsymbol{u}, \boldsymbol{v})=\frac{1}{8}\left[\ln \frac{(\boldsymbol{x}-\boldsymbol{v})^{2}(\boldsymbol{y}-\boldsymbol{u})^{2}}{(\boldsymbol{x}-\boldsymbol{u})^{2}(\boldsymbol{y}-\boldsymbol{v})^{2}}\right]^{2}
$$

The factor of $\alpha_{s}^{2}$ has been extracted from the amplitude but included explicitly in Eq. (2.1), in order to facilitate the perturbative counting for the subsequent expressions. Furthermore, $n_{Y}(\boldsymbol{u}, \boldsymbol{v})$ measures the average number of dipoles with coordinates $(\boldsymbol{u}, \boldsymbol{v})$ in the wavefunction of the hadron as produced after a BFKL evolution over the rapidity $Y$.

At this point, and for later convenience, it is useful to invert Eq. (2.1) and express the dipole density in terms of the scattering amplitude. Applying $\nabla_{\boldsymbol{x}}^{2} \nabla_{\boldsymbol{y}}^{2}$ on Eq. (2.1) and using the property $\nabla_{\boldsymbol{x}}^{2} \ln \boldsymbol{x}^{2}=4 \pi \delta^{(2)}(\boldsymbol{x})$, we obtain

$$
n_{Y}(\boldsymbol{x}, \boldsymbol{y})+n_{Y}(\boldsymbol{y}, \boldsymbol{x})=\frac{4}{g^{4}} \nabla_{\boldsymbol{x}}^{2} \nabla_{\boldsymbol{y}}^{2}\langle T(\boldsymbol{x}, \boldsymbol{y})\rangle_{Y},
$$

with the assumption $\boldsymbol{x} \neq \boldsymbol{y}$.

Now let us consider the scattering of a pair of dipoles $\left(\boldsymbol{x}_{1}, \boldsymbol{y}_{1}\right)$ and $\left(\boldsymbol{x}_{2}, \boldsymbol{y}_{2}\right)$ off the 
target. Clearly the extension of Eq. (2.1) reads

$$
\left\langle T^{(2)}\left(\boldsymbol{x}_{1}, \boldsymbol{y}_{1} ; \boldsymbol{x}_{2}, \boldsymbol{y}_{2}\right)\right\rangle_{Y}=\alpha_{s}^{4} \int_{\boldsymbol{u}_{i}, \boldsymbol{v}_{i}} \mathcal{A}_{0}\left(\boldsymbol{x}_{1}, \boldsymbol{y}_{1} \mid \boldsymbol{u}_{1}, \boldsymbol{v}_{1}\right) \mathcal{A}_{0}\left(\boldsymbol{x}_{2}, \boldsymbol{y}_{2} \mid \boldsymbol{u}_{2}, \boldsymbol{v}_{2}\right) n_{Y}^{(2)}\left(\boldsymbol{u}_{1}, \boldsymbol{v}_{1} ; \boldsymbol{u}_{2}, \boldsymbol{v}_{2}\right)
$$

where $n_{Y}^{(2)}\left(\boldsymbol{u}_{1}, \boldsymbol{v}_{1} ; \boldsymbol{u}_{2}, \boldsymbol{v}_{2}\right)$ is the average number density of pairs of dipoles in the target. As in Ref. [1], we include in $n_{Y}^{(2)}$ only the pairs made of two different dipoles, that is, we do not allow the two external dipoles to scatter off a same dipole in the target. This should be a good approximation so long as the external dipoles have different sizes and/or impact parameters. In particular, if at $Y=0$ the target consists in a single dipole (this will be our initial condition), then $n_{0}^{(2)}=0$, and therefore $\left\langle T^{(2)}\right\rangle_{0}=0$ as well.

The evolution equation for $\left\langle T^{(2)}\right\rangle_{Y}$ can be obtained from the corresponding equation for $n_{Y}^{(2)}$, which has been derived in Ref. [1]. The inhomogeneous part of the latter, i.e. the piece which describes low-density fluctuations, reads

$$
\left.\frac{\partial n_{Y}^{(2)}\left(\boldsymbol{u}_{1}, \boldsymbol{v}_{1} ; \boldsymbol{u}_{2}, \boldsymbol{v}_{2}\right)}{\partial Y}\right|_{\text {fluct }}=\frac{\bar{\alpha}_{s}}{2 \pi} \mathcal{M}\left(\boldsymbol{u}_{1}, \boldsymbol{v}_{2}, \boldsymbol{u}_{2}\right) n_{Y}\left(\boldsymbol{u}_{1}, \boldsymbol{v}_{2}\right) \delta^{(2)}\left(\boldsymbol{u}_{2}-\boldsymbol{v}_{1}\right)+\{1 \leftrightarrow 2\}
$$

with the dipole kernel [10]

$$
\mathcal{M}(\boldsymbol{x}, \boldsymbol{y}, \boldsymbol{z})=\frac{(\boldsymbol{x}-\boldsymbol{y})^{2}}{(\boldsymbol{x}-\boldsymbol{z})^{2}(\boldsymbol{y}-\boldsymbol{z})^{2}}
$$

(The terms not shown here correspond to the usual BFKL evolution of the dipole pair density; see Eq. (5.15) in Ref. [1].) Using Eqs. (2.4) and (2.5) to write the evolution of $\left\langle T^{(2)}\right\rangle_{Y}$ in terms of the dipole density $n_{Y}$ and then employing Eq. (2.3) in order to express $n_{Y}$ in terms of the scattering amplitude $\langle T\rangle_{Y}$, it is straightforward to show that

$$
\begin{gathered}
\left.\frac{\partial\left\langle T^{(2)}\left(\boldsymbol{x}_{1}, \boldsymbol{y}_{1} ; \boldsymbol{x}_{2}, \boldsymbol{y}_{2}\right)\right\rangle_{Y}}{\partial Y}\right|_{\text {fluct }}=\left(\frac{\alpha_{s}}{2 \pi}\right)^{2} \frac{\bar{\alpha}_{s}}{2 \pi} \int_{\boldsymbol{u}, \boldsymbol{v}, \boldsymbol{z}} \mathcal{M}(\boldsymbol{u}, \boldsymbol{v}, \boldsymbol{z}) \mathcal{A}_{0}\left(\boldsymbol{x}_{1}, \boldsymbol{y}_{1} \mid \boldsymbol{u}, \boldsymbol{z}\right) \mathcal{A}_{0}\left(\boldsymbol{x}_{2}, \boldsymbol{y}_{2} \mid \boldsymbol{z}, \boldsymbol{v}\right) \\
\times \nabla_{\boldsymbol{u}}^{2} \nabla_{\boldsymbol{v}}^{2}\langle T(\boldsymbol{u}, \boldsymbol{v})\rangle_{Y}
\end{gathered}
$$

The complete evolution equation for $\left\langle T^{(2)}\right\rangle_{Y}$ can be now obtained by restoring the more standard terms associated with the BFKL evolution and with unitarity corrections at high energy. The former are terms linear in $\left\langle T^{(2)}\right\rangle_{Y}$, while the latter are non-linear terms proportional to the scattering amplitude $\left\langle T^{(3)}\right\rangle_{Y}$ for a system of three external dipoles, and are the same as in the corresponding Balitsky-JIMWLK equation $[2,4,5]$ 
(here, for large $N_{c}$ ). Thus our final evolution equation for $\left\langle T^{(2)}\right\rangle_{Y}$ reads

$$
\begin{aligned}
& \frac{\partial\left\langle T^{(2)}\left(\boldsymbol{x}_{1}, \boldsymbol{y}_{1} ; \boldsymbol{x}_{2}, \boldsymbol{y}_{2}\right)\right\rangle_{Y}}{\partial Y}=\frac{\bar{\alpha}_{s}}{2 \pi} \int_{\boldsymbol{z}}\left\{\left[\mathcal{M}_{\boldsymbol{x}_{1} \boldsymbol{y}_{1} \boldsymbol{z}} \otimes\left\langle T^{(2)}\left(\boldsymbol{x}_{1}, \boldsymbol{y}_{1} ; \boldsymbol{x}_{2}, \boldsymbol{y}_{2}\right)\right\rangle_{Y}\right.\right. \\
& \left.\left.-\mathcal{M}\left(\boldsymbol{x}_{1}, \boldsymbol{y}_{1}, \boldsymbol{z}\right)\left\langle T^{(3)}\left(\boldsymbol{x}_{1}, \boldsymbol{z} ; \boldsymbol{z}, \boldsymbol{y}_{1} ; \boldsymbol{x}_{2}, \boldsymbol{y}_{2}\right)\right\rangle_{Y}\right]+[1 \leftrightarrow 2]\right\} \\
& +\left.\frac{\partial\left\langle T^{(2)}\left(\boldsymbol{x}_{1}, \boldsymbol{y}_{1} ; \boldsymbol{x}_{2}, \boldsymbol{y}_{2}\right)\right\rangle_{Y}}{\partial Y}\right|_{\text {fluct }},
\end{aligned}
$$

with the last term given by Eq. (2.7) and where we have introduced the shorthand notation

$$
\mathcal{M}_{\boldsymbol{x} \boldsymbol{y} \boldsymbol{z}} \otimes f(\boldsymbol{x}, \boldsymbol{y}) \equiv \mathcal{M}(\boldsymbol{x}, \boldsymbol{y}, \boldsymbol{z})[-f(\boldsymbol{x}, \boldsymbol{y})+f(\boldsymbol{x}, \boldsymbol{z})+f(\boldsymbol{z}, \boldsymbol{y})]
$$

Under the same approximations, the scattering amplitude for a single dipole obeys the following equation, which is formally the same as the first equation in Balitsky hierarchy:

$$
\frac{\partial\langle T(\boldsymbol{x}, \boldsymbol{y})\rangle_{Y}}{\partial Y}=\frac{\bar{\alpha}_{s}}{2 \pi} \int_{\boldsymbol{z}}\left\{\mathcal{M}_{\boldsymbol{x} \boldsymbol{y} \boldsymbol{z}} \otimes\left\langle T(\boldsymbol{x}, \boldsymbol{y}\rangle_{Y}-\mathcal{M}(\boldsymbol{x}, \boldsymbol{y}, \boldsymbol{z})\left\langle T^{(2)}(\boldsymbol{x}, \boldsymbol{z} ; \boldsymbol{z}, \boldsymbol{y})\right\rangle_{Y}\right\}\right.
$$

Note that the evolutions of $\langle T\rangle_{Y}$ and $\left\langle T^{(2)}\right\rangle_{Y}$ are coupled by the fluctuation term in Eq. (2.8), in agreement with [1]. Moreover, $\left\langle T^{(2)}\right\rangle_{Y}$ is also coupled to $\left\langle T^{(3)}\right\rangle_{Y}$, by the unitarity corrections, so the equations above do not close by themselves, but rather are a part of an extended (actually, infinite) hierarchy. The higher equations in this hierarchy will be written down in the next section.

Before closing this section, we would like to comment on the simplified version of Eq. (2.7) which was proposed in [1]. There, an approximation was done at the level of Eq. (2.2), in which the non-locality of the 2-gluon exchange interaction was neglected. That is, the elementary dipole-dipole scattering amplitude was effectively assumed to be

$$
\alpha_{s}^{2} \mathcal{A}_{0}(\boldsymbol{x}, \boldsymbol{y} \mid \boldsymbol{u}, \boldsymbol{v}) \simeq c \alpha_{s}^{2}(\boldsymbol{x}-\boldsymbol{y})^{4} \delta^{(2)}(\boldsymbol{x}-\boldsymbol{u}) \delta^{(2)}(\boldsymbol{y}-\boldsymbol{v})
$$

where $c$ is an unknown "fudge" factor. Then, by following the same steps as above, it was shown that the right hand side of Eq. (2.7) is

$$
c \alpha_{s}^{2} \frac{\bar{\alpha}_{s}}{2 \pi} \frac{\left(\boldsymbol{x}_{1}-\boldsymbol{y}_{1}\right)^{2}\left(\boldsymbol{x}_{2}-\boldsymbol{y}_{2}\right)^{2}}{\left(\boldsymbol{x}_{1}+\boldsymbol{y}_{2}\right)^{2}}\left\langle T\left(\boldsymbol{x}_{1}, \boldsymbol{y}_{2}\right)\right\rangle_{Y} \delta^{(2)}\left(\boldsymbol{x}_{2}-\boldsymbol{y}_{1}\right)+\{1 \leftrightarrow 2\} .
$$

After a coarse-graining in impact parameter space and a saddle point approximation, a Langevin equation, equivalent to the stochastic FKPP equation, was finally obtained. However, these equations suffer from the shortcomings mentioned in the Introduction. 


\section{The Langevin Equation}

In order to have a complete hierarchy of equations, we need to generalize Eq. (2.7) to the case where an arbitrary number of dipoles, say $\kappa$, scatters of the target. Given the $\kappa$-th density of dipoles $n_{Y}\left(\boldsymbol{u}_{1}, \boldsymbol{v}_{1} ; \ldots ; \boldsymbol{u}_{\kappa}, \boldsymbol{v}_{\kappa}\right) \equiv n_{Y}(\{\boldsymbol{u}, \boldsymbol{v}\})$ in the wavefunction of the hadron, the amplitude for $\kappa$-dipoles scattering reads

$$
\left\langle T^{(\kappa)}(\{\boldsymbol{x}, \boldsymbol{y}\})\right\rangle_{Y}=\alpha_{s}^{2 \kappa} \int_{\boldsymbol{u}_{i}, \boldsymbol{v}_{i}} \mathcal{A}_{0}\left(\boldsymbol{x}_{1}, \boldsymbol{y}_{1} \mid \boldsymbol{u}_{1}, \boldsymbol{v}_{1}\right) \ldots \mathcal{A}_{0}\left(\boldsymbol{x}_{\kappa}, \boldsymbol{y}_{\kappa} \mid \boldsymbol{u}_{\kappa}, \boldsymbol{v}_{\kappa}\right) n_{Y}^{(\kappa)}(\{\boldsymbol{u}, \boldsymbol{v}\}) .
$$

The corresponding evolution equation is obtained by the straightforward generalization of the procedure that we followed in the previous section. The rate of change, due to fluctuations, of the $\kappa$-th density of dipoles of the hadronic wavefunction depends only in the $(\kappa-1)$-th density, and therefore the same will be true for the scattering amplitude $\left\langle T^{(\kappa)}(\{\boldsymbol{x}, \boldsymbol{y}\})\right\rangle_{Y}$, since Eq. (3.1) relates quantities of the "same order" in $\kappa$. The ensuing equation reads

$$
\begin{array}{r}
\left.\frac{\partial\left\langle T^{(\kappa)}(\{\boldsymbol{x}, \boldsymbol{y}\})\right\rangle_{Y}}{\partial Y}\right|_{\text {fluct }}=\left(\frac{\alpha_{s}}{2 \pi}\right)^{2} \frac{\bar{\alpha}_{s}}{2 \pi} \int_{\boldsymbol{u}, \boldsymbol{v}, \boldsymbol{z}} \mathcal{M}(\boldsymbol{u}, \boldsymbol{v}, \boldsymbol{z}) \mathcal{A}_{0}\left(\boldsymbol{x}_{1}, \boldsymbol{y}_{1} \mid \boldsymbol{u}, \boldsymbol{z}\right) \mathcal{A}_{0}\left(\boldsymbol{x}_{2}, \boldsymbol{y}_{2} \mid \boldsymbol{z}, \boldsymbol{v}\right) \\
\times \nabla_{\boldsymbol{u}}^{2} \nabla_{\boldsymbol{v}}^{2}\left\langle T^{(\kappa-1)}\left(\boldsymbol{u}, \boldsymbol{v} ; \boldsymbol{x}_{3}, \boldsymbol{y}_{3} ; \ldots ; \boldsymbol{x}_{\kappa}, \boldsymbol{y}_{\kappa}\right)\right\rangle_{Y}+\text { perm. }
\end{array}
$$

where "perm." stands for all possible permutations in the arguments of $\left\langle T^{(\kappa-1)}\right\rangle_{Y}$, so that the total number of terms in the right hand side is $\kappa(\kappa-1) / 2$.

It is now straightforward to add to the r.h.s. of Eq. (3.2) the (large $-N_{c}$ version of the) standard Balitsky-JIMWLK terms, and thus obtain the complete hierarchy for $\left\langle T^{(\kappa)}\right\rangle_{Y}$ within the present approximation. Since in practice (e.g., for numerical studies) it seems difficult to deal with a hierarchy as a whole, it is convenient to notice that one can equivalently reformulate this hierarchy as a single stochastic equation. Let us consider the following Langevin equation

$$
\left.\frac{\partial T_{Y}(\boldsymbol{x}, \boldsymbol{y})}{\partial Y}\right|_{\text {fluct }}=\frac{\alpha_{s}}{2 \pi} \sqrt{\frac{\bar{\alpha}_{s}}{2 \pi}} \int_{\boldsymbol{u}, \boldsymbol{v}, \boldsymbol{z}} \mathcal{A}_{0}(\boldsymbol{x}, \boldsymbol{y} \mid \boldsymbol{u}, \boldsymbol{z}) \frac{|\boldsymbol{u}-\boldsymbol{v}|}{(\boldsymbol{u}-\boldsymbol{z})^{2}} \sqrt{\nabla_{\boldsymbol{u}}^{2} \nabla_{\boldsymbol{v}}^{2} T_{Y}(\boldsymbol{u}, \boldsymbol{v})} \nu(\boldsymbol{u}, \boldsymbol{v}, \boldsymbol{z}, Y),
$$

where the noise satisfies

$$
\left\langle\nu\left(\boldsymbol{u}_{1}, \boldsymbol{v}_{1}, \boldsymbol{z}_{1}, Y\right) \nu\left(\boldsymbol{u}_{2}, \boldsymbol{v}_{2}, \boldsymbol{z}_{2}, Y^{\prime}\right)\right\rangle=\delta^{(2)}\left(\boldsymbol{u}_{1}-\boldsymbol{v}_{2}\right) \delta^{(2)}\left(\boldsymbol{v}_{1}-\boldsymbol{u}_{2}\right) \delta^{(2)}\left(\boldsymbol{z}_{1}-\boldsymbol{z}_{2}\right) \delta\left(Y-Y^{\prime}\right)
$$

with all the other correlators being zero. Note that the correlator (3.4) is non-diagonal in the first two arguments $(\boldsymbol{u}, \boldsymbol{v})$ of the noise. A similar property has been recently noticed

in Ref. [28], in a physical context which is very close to ours (see the discussion in Sect. 4 
below), but we are not aware about previous studies of such a non-diagonal noise in other (physical or mathematical) contexts. The stochastic problem above must be understood with the Ito prescription for rapidity discretization (see, e.g., [1,29] for details). Then one can check that by solving this equation one generates the same correlations as from the original hierarchy in Eq. (3.2). Indeed, consider the change in the $\kappa$-dipole function $T_{1} \ldots T_{\kappa}$ under an increase $\Delta Y$ in rapidity (in $T_{i}$, the index $i$ serves as a shorthand for the coordinates of the $i$-th dipole). Eqs. (3.3) and (3.4) imply that $\Delta T \sim \sqrt{\Delta Y}$, so in evaluating $\Delta\left(T_{1} \ldots T_{\kappa}\right)$, it is enough to keep terms of second order in the $\Delta T$ 's :

$$
\Delta\left(T_{1} \ldots T_{\kappa}\right)=\left(T_{1}+\Delta T_{1}\right) \ldots\left(T_{\kappa}+\Delta T_{\kappa}\right)-T_{1} \ldots T_{\kappa} \rightarrow \Delta T_{1} \Delta T_{2} T_{3} \ldots T_{\kappa}+\text { perm }
$$

Indeed, the linear terms will vanish after averaging over the noise, whereas the cubic terms, or higher, will be of higher order in $\Delta Y$, and thus will vanish in the continuum limit $\Delta Y \rightarrow 0$. The transverse coordinates dependence of the noise correlation in Eq. (3.4) is such that, when we take the expectation value in Eq. (3.5), the emerging hierarchy is exactly the same as the one in Eq. (3.2), provided we make the identification $\left\langle T_{1} \ldots T_{\kappa}\right\rangle \leftrightarrow$ $\left\langle T^{(\kappa)}(\{\boldsymbol{x}, \boldsymbol{y}\})\right\rangle_{Y}$

Eq. (3.3) refers solely to the fluctuations terms in the hierarchy, but the standard Balitsky-JIMWLK terms can be easily added [1]. We finally obtain

$$
\begin{aligned}
\frac{\partial T_{Y}(\boldsymbol{x}, \boldsymbol{y})}{\partial Y}= & \frac{\bar{\alpha}_{s}}{2 \pi} \int_{\boldsymbol{z}}\left[\mathcal{M}_{\boldsymbol{x y} \boldsymbol{z}} \otimes T_{Y}(\boldsymbol{x}, \boldsymbol{y})-\mathcal{M}(\boldsymbol{x}, \boldsymbol{y}, \boldsymbol{z}) T_{Y}(\boldsymbol{x}, \boldsymbol{z}) T_{Y}(\boldsymbol{z}, \boldsymbol{y})\right] \\
& +\left.\frac{\partial T_{Y}(\boldsymbol{x}, \boldsymbol{y})}{\partial Y}\right|_{\text {fluct }},
\end{aligned}
$$

with the last term being given by Eq. (3.3). This equation together with the noise term (3.4) is the Langevin problem equivalent to the non-linear evolution equations with pomeron loops. But, as mentioned above, the non-diagonal nature of the noise correlator (3.4) is a rather unusual mathematical property, and presently we do not know whether such a noise can be useful for numerical studies, or not.

\section{The Color Glass Condensate Approach}

An alternative approach to high-energy QCD is the Color Glass Condensate (CGC) formalism in which one studies the random color fields created by 'color sources' (gluons or dipoles) within the target, and the evolution of the respective correlation functions with increasing $Y$ (see [30] for review papers and more references). In a specific gauge, the color field in the target has only one component, the light-cone component $A_{a}^{+}$, which is traditionally denoted as $A_{a}^{+}(x) \equiv \alpha^{a}\left(x^{-}, \boldsymbol{x}\right)$. (This is a function of the longitudinal coordinate $x^{-}$together with the transverse coordinate $\boldsymbol{x}$.) Let $W_{Y}[\alpha]$ represent the functional probability distribution for finding a particular configuration of this field at rapidity $Y$. 
Then the expectation value of an operator $\mathcal{O}[\alpha]$ is given by

$$
\langle\mathcal{O}[\alpha]\rangle_{Y}=\int D \alpha W_{Y}[\alpha] \mathcal{O}[\alpha]
$$

For example the operator for the scattering between a single dipole $(\boldsymbol{x}, \boldsymbol{y})$ and the CGC is computed in the eikonal approximation as

$$
T(\boldsymbol{x}, \boldsymbol{y})=1-\frac{1}{N_{c}} \operatorname{tr}\left(V_{\boldsymbol{x}}^{\dagger} V_{\boldsymbol{y}}\right)
$$

where $V_{\boldsymbol{x}}^{\dagger}$ is a Wilson line describing the scattering between the quark and the color field in the CGC (below, $\mathcal{P}$ stands for a path ordered product),

$$
V_{\boldsymbol{x}}^{\dagger}[\alpha]=\mathcal{P} \exp \left[i g \int \mathrm{d} x^{-} \alpha^{a}\left(x^{-}, \boldsymbol{x}\right) t^{a}\right]
$$

while $V_{\boldsymbol{y}}$ refers similarly to the antiquark. Furthermore, the corresponding operator for a dipole pair is $T^{(2)}\left(\boldsymbol{x}_{1}, \boldsymbol{y}_{1} ; \boldsymbol{x}_{2}, \boldsymbol{y}_{2}\right)=T\left(\boldsymbol{x}_{1}, \boldsymbol{y}_{1}\right) T\left(\boldsymbol{x}_{1}, \boldsymbol{y}_{1}\right)$, with $T$ given by Eq. (4.2).

Under an increase $\mathrm{d} Y$ in rapidity, the correlation functions of the color field change because of the induced emission of a small- $x$ gluon. These changes can be accommodated in a functional evolution equation for the probability distribution, which can be written in Hamiltonian form (at least, formally) :

$$
\frac{\partial}{\partial Y} W_{Y}[\alpha]=H W_{Y}[\alpha]
$$

Then the evolution equation for $\langle\mathcal{O}[\alpha]\rangle_{Y}$ can be deduced from Eqs. (4.1) and (4.4).

The original version of the Hamiltonian which enters Eq. (4.4), known as the JIMWLK Hamiltonian $\left(H_{\text {JIMwLK }}\right.$ ) [3-5], leads to Balitsky equations [2] when applied to scattering operators like (4.2). $H_{\text {JIMwLK }}$ is a second-order functional differential operator, with a kernel which is non-linear in the field $\alpha$ to all orders. The quadratic part of this kernel is responsible for the linear, BFKL, evolution, while the terms of higher order in $\alpha$ generate non-linear terms, so like the terms expressing unitarity corrections in Balitsky equations. As we have noticed in [1], the evolution generated by $H_{\text {JIMWLK }}$ does not include gluon splitting, and therefore cannot describe the correlations associated with fluctuations in the gluon number, which are however important in the dilute regime. Very recently, Mueller, Shoshi and Wong [28] have proposed an additional term $H_{\text {fluct }}$, which is inspired by the probabilistic interpretation of the evolution in the dipole model [10,31], and which takes into account the low density fluctuations at the same level of accuracy as the dipole picture. Thus, the total Hamiltonian used in Ref. [28] can be written as:

$$
H=H_{\text {JIMWLK }}+H_{\text {fluct }} \text {, }
$$


where $H_{\text {JIMwLK }}$ can be found in Refs. $[4,5]$ (see also [32] for a simple expression), and

$$
\begin{aligned}
H_{\text {fluct }}=-\frac{\pi \alpha_{s}}{4 N_{c}^{3}} \frac{\bar{\alpha}_{s}}{2 \pi} \int & \mathcal{M}(\boldsymbol{u}, \boldsymbol{v}, \boldsymbol{z}) \mathcal{G}\left(\boldsymbol{u}_{1} \mid \boldsymbol{u}, \boldsymbol{z}\right) \mathcal{G}\left(\boldsymbol{v}_{1} \mid \boldsymbol{u}, \boldsymbol{z}\right) \mathcal{G}\left(\boldsymbol{u}_{2} \mid \boldsymbol{z}, \boldsymbol{v}\right) \mathcal{G}\left(\boldsymbol{v}_{2} \mid \boldsymbol{z}, \boldsymbol{v}\right) \\
& \times \frac{\delta}{\delta \alpha^{a}\left(\boldsymbol{u}_{1}\right)} \frac{\delta}{\delta \alpha^{a}\left(\boldsymbol{v}_{1}\right)} \frac{\delta}{\delta \alpha^{b}\left(\boldsymbol{u}_{2}\right)} \frac{\delta}{\delta \alpha^{b}\left(\boldsymbol{v}_{2}\right)} \nabla_{\boldsymbol{u}}^{2} \nabla_{\boldsymbol{v}}^{2} \alpha^{c}(\boldsymbol{u}) \alpha^{c}(\boldsymbol{v}) .
\end{aligned}
$$

Here, the integration goes over all the transverse coordinates $\boldsymbol{u}, \boldsymbol{v}, \boldsymbol{z}, \boldsymbol{u}_{1}, \boldsymbol{v}_{1}, \boldsymbol{u}_{2}, \boldsymbol{v}_{2}$, and $\alpha^{a}(\boldsymbol{x}) \equiv \int d x^{-} \alpha^{a}\left(x^{-}, \boldsymbol{x}\right)$. The function $\mathcal{G}\left(\boldsymbol{u}_{1} \mid \boldsymbol{u}, \boldsymbol{z}\right)$ is, up to a factor $g t^{a}$, the classical field created by the elementary dipole $(\boldsymbol{u}, \boldsymbol{z})$, and reads

$$
\mathcal{G}\left(\boldsymbol{u}_{1} \mid \boldsymbol{u}, \boldsymbol{z}\right)=\frac{1}{4 \pi} \ln \frac{\left(\boldsymbol{u}_{1}-\boldsymbol{z}\right)^{2}}{\left(\boldsymbol{u}_{1}-\boldsymbol{u}\right)^{2}}
$$

In what follows we would like to show that the Hamiltonian (4.6) leads precisely to our previous hierarchy (3.2) for the dipole scattering amplitudes. We shall demonstrate that only for the case of two external dipoles $(\kappa=2)$, since the corresponding proof for arbitrary $\kappa$ follows trivially. Also we shall focus on the contribution induced by the fluctuation piece $H_{\text {fluct }}$ of the Hamiltonian, since the corresponding contribution of $H_{\text {JIMWLK }}$ is well-known $[4,5,29,32]$ to be the respective Balitsky equation. Thus, we start with (cf. Eqs. (4.1) and (4.4)):

$$
\left.\frac{\partial\left\langle T^{(2)}\left(\boldsymbol{x}_{1}, \boldsymbol{y}_{1} ; \boldsymbol{x}_{2}, \boldsymbol{y}_{2}\right)\right\rangle_{Y}}{\partial Y}\right|_{\text {fluct }}=\int D \alpha T^{(2)}\left(\boldsymbol{x}_{1}, \boldsymbol{y}_{1} ; \boldsymbol{x}_{2}, \boldsymbol{y}_{2}\right) H_{\text {fluct }} W_{Y}[\alpha]
$$

In the weak field limit the scattering amplitude is determined from the small coupling expansion of the corresponding Wilson lines operator and it reads

$$
T^{(2)}\left(\boldsymbol{x}_{1}, \boldsymbol{y}_{1} ; \boldsymbol{x}_{2}, \boldsymbol{y}_{2}\right)=\frac{g^{4}}{16 N_{c}^{2}}\left[\alpha^{d}\left(\boldsymbol{x}_{1}\right)-\alpha^{d}\left(\boldsymbol{y}_{1}\right)\right]^{2}\left[\alpha^{e}\left(\boldsymbol{x}_{2}\right)-\alpha^{e}\left(\boldsymbol{y}_{2}\right)\right]^{2}
$$

By partial integrations within the r.h.s. of Eq. (4.8), one can bring the functional differential operator $(\delta / \delta \alpha)^{4}$ (cf. Eq. (4.6)) to act on $T^{(2)}$. This operation annihilates the $\alpha$ fields manifest in Eq. (4.9) and gives rise to a sum of products of two-dimensional delta functions. For consistency with the derivation of Eq. (4.6) [28], we should restrict ourselves to the terms of leading order in $N_{c}$, which arise when a pair of derivatives which are traced over color acts on a pair of fields which carry both the same color indices. Then we can perform the integrations over $\boldsymbol{u}_{1}, \boldsymbol{v}_{1}, \boldsymbol{u}_{2}, \boldsymbol{v}_{2}$, to finally arrive at

$$
\begin{gathered}
\left.\frac{\partial\left\langle T^{(2)}\left(\boldsymbol{x}_{1}, \boldsymbol{y}_{1} ; \boldsymbol{x}_{2}, \boldsymbol{y}_{2}\right)\right\rangle_{Y}}{\partial Y}\right|_{\text {fluct }}=\frac{\alpha_{s}^{3}}{4 \pi N_{c}} \frac{\bar{\alpha}_{s}}{2 \pi} \int_{\boldsymbol{u}, \boldsymbol{v}, \boldsymbol{z}} \mathcal{M}(\boldsymbol{u}, \boldsymbol{v}, \boldsymbol{z}) \mathcal{A}_{0}\left(\boldsymbol{x}_{1}, \boldsymbol{y}_{1} \mid \boldsymbol{u}, \boldsymbol{z}\right) \mathcal{A}_{0}\left(\boldsymbol{x}_{2}, \boldsymbol{y}_{2} \mid \boldsymbol{z}, \boldsymbol{v}\right) \\
\times \nabla_{\boldsymbol{u}}^{2} \nabla_{\boldsymbol{v}}^{2}\left\langle-2 \alpha^{c}(\boldsymbol{u}) \alpha^{c}(\boldsymbol{v})\right\rangle_{Y} .
\end{gathered}
$$


The last factor in the above equation may be rewritten as

$$
\nabla_{\boldsymbol{u}}^{2} \nabla_{\boldsymbol{v}}^{2}\left\langle-2 \alpha^{c}(\boldsymbol{u}) \alpha^{c}(\boldsymbol{v})\right\rangle_{Y}=\nabla_{\boldsymbol{u}}^{2} \nabla_{\boldsymbol{v}}^{2}\left\langle\left[\alpha^{c}(\boldsymbol{u})-\alpha^{c}(\boldsymbol{v})\right]^{2}\right\rangle_{Y}=\frac{N_{c}}{\pi \alpha_{s}} \nabla_{\boldsymbol{u}}^{2} \nabla_{\boldsymbol{v}}^{2}\langle T(\boldsymbol{u}, \boldsymbol{v})\rangle_{Y}
$$

where we have also used the weak-field expansion of the scattering amplitude (4.2) for a single dipole. With this identification, Eq. (4.10) leads indeed to Eq. (2.7), which completes our proof.

\section{The triple pomeron vertex}

The above derivation of our new equations within the CGC approach sheds more light on their physical interpretation and confirms the original discussion in Ref. [1]: when the evolution is included in the target wavefunction, the fluctuation terms in the equations for the amplitudes (e.g., the term linear in $\left\langle T^{(\kappa-1)}\right\rangle_{Y}$ in the r.h.s. of Eq. (3.2) for $\left\langle T^{(\kappa)}\right\rangle_{Y}$ ) correspond to gluon splittings, by which we mean more precisely the transition vertex from two to four gluons described by the new piece $H_{\text {fluct }}$, Eq. (4.6), of the CGC Hamiltonian. Similarly, the non-linear terms responsible for unitarization (e.g., the term $\left\langle T^{(3)}\right\rangle_{Y}$ in the r.h.s. of Eq. (2.8) for $\left\langle T^{(2)}\right\rangle_{Y}$ ), correspond to gluon mergings, as described by the terms of higher order in $\alpha$ in the original JIMWLK Hamiltonian. By 'gluon mergings' we mean all the vertices leading to a reduction in the number of gluon fields. The simplest among them is the transition from four to two gluons, and is described by that term in $H_{\text {JIMWLK }}$ which is dual to Eq. (4.6), in the sense of containing four powers of $\alpha$ and two powers of the derivative $(\delta / \delta \alpha)$. In general, one would expect this duality to extend to higher vertices; that is, $H_{\text {fluct }}$ is expected to include terms with higher-order derivatives, which should be 'dual' to the terms with higher powers of $\alpha$ in $H_{\text {JIMwLK }}$. Since such terms do not appear to be generated by the dipole picture which has been used to study fluctuations so far $[1,6,7,28]$, they are presumably of higher order in $1 / N_{c}$.

Furthermore, within the framework of the large $-N_{c}$ approximation, the gluon number changing transitions alluded to above should be tantamount to pomeron splittings, or mergings, i.e., processes through which one BFKL pomeron is connected to two. For the non-linear term in the Balitsky-Kovchegov equation [2,33] (and thus, by extension, for the whole Balitsky hierarchy at large $N_{c}$ ), this has been checked already in Refs. $[25,27]$. Namely, it has been shown there that the general 2-to-4 gluon vertex [21] reduces essentially to the dipole kernel, Eq. (2.6), when restricted to the large $-N_{c}$ limit and to the physical conditions appropriate for the merging of two pomerons ${ }^{2}$. This is indeed consistent with the structure of the non-linear term in Eq. (3.6).

2 Namely, the four gluons on one side of the vertex are pairwise connected in color singlet states, which moreover are multiplied by functions which vanish at equal coordinates, so like $T_{Y}(\boldsymbol{x}, \boldsymbol{z}) T_{Y}(\boldsymbol{z}, \boldsymbol{y})$ in the r.h.s. of Eq. (3.6). 
In what follows, we shall present a similar check for the vertex that enters the fluctuation term in Eq. (2.7) (and thus the noise term in Eq. (3.6)). To that aim, we shall compare our result for this vertex with that in Ref. [23], where a linear evolution equation has been constructed which includes the triple-pomeron vertex for one pomeron splitting into two. (The equation written in Ref. [23] refers to the diffractive amplitude for four gluons, which is quite similar to the scattering amplitude for two dipoles in our approach.)

From our equations, the amputated version of the fluctuation vertex can be immediately extracted, as the function which is convoluted with $\langle T(\boldsymbol{u}, \boldsymbol{v})\rangle_{Y}$ in the r.h.s. of Eq. (2.7). But since in Ref. [23] one rather finds the non-amputated version of the triplepomeron vertex (this is referred there as the 'triple pomeron interaction', or TPI), we find it convenient to compute the similar quantity in our approach as well, by solving the linearized version of Eq. (2.8). Assuming the initial condition $\left\langle T^{(2)}\right\rangle_{0}=0$, the solution to this inhomogeneous equation can be written as

$$
\begin{gathered}
\left\langle T^{(2)}\left(\boldsymbol{x}_{1}, \boldsymbol{y}_{1} ; \boldsymbol{x}_{2}, \boldsymbol{y}_{2}\right)\right\rangle_{Y}=\int_{0}^{Y} \mathrm{~d} y \int_{\boldsymbol{u}_{i}, \boldsymbol{v}_{i}} G_{Y-y}\left(\boldsymbol{x}_{1}, \boldsymbol{y}_{1} \mid \boldsymbol{u}_{1}, \boldsymbol{v}_{1}\right) G_{Y-y}\left(\boldsymbol{x}_{2}, \boldsymbol{y}_{2} \mid \boldsymbol{u}_{2}, \boldsymbol{v}_{2}\right) \\
\times\left.\frac{\partial\left\langle T^{(2)}\left(\boldsymbol{u}_{1}, \boldsymbol{v}_{1} ; \boldsymbol{u}_{2}, \boldsymbol{v}_{2}\right)\right\rangle_{y}}{\partial y}\right|_{\text {fluct }},
\end{gathered}
$$

where the Green's function $G_{Y}$ satisfies the BFKL equation (which is homogeneous)

$$
\left[\frac{\partial}{\partial Y}-\frac{\bar{\alpha}_{s}}{2 \pi} \int_{\boldsymbol{z}} \mathcal{M}_{\boldsymbol{x} \boldsymbol{y} \boldsymbol{z}} \otimes\right] G_{Y}(\boldsymbol{x}, \boldsymbol{y} \mid \boldsymbol{u}, \boldsymbol{v})=0
$$

along with the initial condition

$$
G_{Y}(\boldsymbol{x}, \boldsymbol{y} \mid \boldsymbol{u}, \boldsymbol{v})=\delta^{(2)}(\boldsymbol{x}-\boldsymbol{u}) \delta^{(2)}(\boldsymbol{y}-\boldsymbol{v})
$$

Instead of directly solving Eqs. (5.2) and (5.3), which is a simple task in any case, it is more convenient for our purposes to observe that one can combine each of the Green's function in Eq. (5.1) with one of the elementary amplitudes $\mathcal{A}_{0}$ contained in the source term $\partial\left\langle T^{(2)}\right\rangle_{y} / \partial y$, cf. Eq. (2.7). By doing this, we can reconstruct the dipole-dipole scattering amplitude in the BFKL approximation (the particular case of the amplitude $\langle T(\boldsymbol{x}, \boldsymbol{y})\rangle_{Y}$ in which the target is itself an elementary dipole). Indeed, since the dipole-dipole scattering amplitude in the linear regime satisfies the BFKL equation, one can write

$$
\mathcal{A}_{Y-y}\left(\boldsymbol{x}_{1}, \boldsymbol{y}_{1} \mid \boldsymbol{u}, \boldsymbol{z}\right)=\int_{\boldsymbol{u}_{1}, \boldsymbol{v}_{1}} G_{Y-y}\left(\boldsymbol{x}_{1}, \boldsymbol{y}_{1} \mid \boldsymbol{u}_{1}, \boldsymbol{v}_{1}\right) \mathcal{A}_{0}\left(\boldsymbol{u}_{1}, \boldsymbol{y}_{1} \mid \boldsymbol{u}, \boldsymbol{z}\right)
$$

along with an analogous equation for the $\boldsymbol{u}_{2}, \boldsymbol{v}_{2}$ integration. Therefore, we can finally 
express the scattering amplitude for the dipole pair in the form

$$
\begin{array}{r}
\left\langle T^{(2)}\left(\boldsymbol{x}_{1}, \boldsymbol{y}_{1} ; \boldsymbol{x}_{2}, \boldsymbol{y}_{2}\right)\right\rangle_{Y}=\left(\frac{\alpha_{s}}{2 \pi}\right)^{2} \frac{\bar{\alpha}_{s}}{2 \pi} \int_{0}^{Y} \mathrm{~d} y \int_{\substack{\boldsymbol{u}, \boldsymbol{v}, \boldsymbol{z}\\
}} \mathcal{M}(\boldsymbol{u}, \boldsymbol{v}, \boldsymbol{z}) \mathcal{A}_{Y-y}\left(\boldsymbol{x}_{1}, \boldsymbol{y}_{1} \mid \boldsymbol{u}, \boldsymbol{z}\right) \\
\quad \times \mathcal{A}_{Y-y}\left(\boldsymbol{x}_{2}, \boldsymbol{y}_{2} \mid \boldsymbol{z}, \boldsymbol{v}\right) \nabla_{\boldsymbol{u}}^{2} \nabla_{\boldsymbol{v}}^{2}\langle T(\boldsymbol{u}, \boldsymbol{v})\rangle_{y},
\end{array}
$$

which is indeed recognized as the 'triple pomeron interaction' in [23] (see Eq. (69) there). This concludes our present analysis.

Only a few days ago, when this work was already completed, a preprint appeared by E. Levin and M. Lublinsky [34] in which a hierarchy of equations including pomeron loops is proposed. Although the general structure of this hierarchy bears some similarity to ours, its derivation and detailed form remain different.

\section{Acknowledgments}

We would like to thank our colleagues Yoshitaka Hatta, Larry McLerran, Al Mueller, Arif Shoshi, Anna Stasto and Stephen Wong for many useful, and critical, comments on our previous preprint which have inspired some of the developments presented here.

\section{References}

[1] E. Iancu and D.N. Triantafyllopoulos, "A Langevin equation for high energy evolution with Pomeron Loops", arXiv:hep-ph/0411405.

[2] I. Balitsky, Nucl. Phys. B463 (1996) 99; Phys. Rev. Lett. 81 (1998) 2024; Phys. Lett. B518 (2001) 235; High-energy QCD and Wilson lines, arXiv:hep-ph/0101042.

[3] J. Jalilian-Marian, A. Kovner, A. Leonidov and H. Weigert, Nucl. Phys. B504 (1997) 415; Phys. Rev. D59 (1999) 014014.

[4] E. Iancu, A. Leonidov and L. McLerran, Nucl. Phys. A692 (2001) 583; Phys. Lett. B510 (2001) 133; E. Ferreiro, E. Iancu, A. Leonidov and L. McLerran, Nucl. Phys. A703 (2002) 489.

[5] H. Weigert, Nucl. Phys. A703 (2002) 823.

[6] A.H. Mueller and A.I. Shoshi, Nucl. Phys. B692 (2004) 175.

[7] E. Iancu, A.H. Mueller, and S. Munier, Phys. Lett. B606 (2005) 342.

[8] L.V. Gribov, E.M. Levin, and M.G. Ryskin, Phys. Rept. 100 (1983) 1. 
[9] L. McLerran and R. Venugopalan, Phys. Rev. D49 (1994) 2233; ibid. 49 (1994) 3352; ibid. 50 (1994) 2225.

[10] A.H. Mueller, Nucl. Phys. B415 (1994) 373; A.H. Mueller and B. Patel, Nucl. Phys. B425 (1994) 471.

[11] A.H. Mueller, Nucl. Phys. B437 (1995) 107.

[12] G.P. Salam, Nucl. Phys. B449 (1995) 589; Nucl. Phys. B461 (1996) 512.

[13] L.N. Lipatov, Sov. J. Nucl. Phys. 23 (1976) 338;

E.A. Kuraev, L.N. Lipatov and V.S. Fadin, Zh. Eksp. Teor. Fiz 72, 3 (1977) (Sov. Phys. JETP 45 (1977) 199);

Ya.Ya. Balitsky and L.N. Lipatov, Sov. J. Nucl. Phys. 28 (1978) 822.

[14] E. Iancu, K. Itakura, and L. McLerran, Nucl. Phys. A708 (2002) 327.

[15] A.H. Mueller and D.N. Triantafyllopoulos, Nucl. Phys. B640 (2002) 331.

[16] D.N. Triantafyllopoulos, Nucl. Phys. B648 (2003) 293.

[17] S. Munier and R. Peschanski, Phys. Rev. Lett. 91 (2003) 232001; Phys. Rev. D69 (2004) 034008; ibid. D70 (2004) 077503.

[18] V.N. Gribov and L.N. Lipatov, Sov. Journ. Nucl. Phys. 15 (1972), 438; G. Altarelli and G. Parisi, Nucl. Phys. B126 (1977), 298; Yu. L. Dokshitzer, Sov. Phys. JETP 46 (1977), 641.

[19] A.M. Stasto, private communication.

[20] For a recent review, see W. Van Saarloos, Phys. Rep. 386 (2003) 29.

[21] J. Bartels and M. Wüsthoff, Z. Phys. C66 (1995) 157.

[22] R. Peschanski, Phys. Lett. B409 (1997) 491.

[23] M. Braun and G.P. Vacca, Eur. Phys. J. C6 (1999) 147.

[24] J. Bartels and C. Ewerz, JHEP 9909 (1999) 026;

C. Ewerz and V. Schatz, Nucl. Phys. A736 (2004) 371.

[25] J. Bartels, L.N. Lipatov and G.P. Vacca, Nucl. Phys. B706 (2005) 391.

[26] J. Bartels, M. Braun and G.P. Vacca, "Pomeron vertices in perturbative QCD in diffractive scattering", arXiv:hep-ph/0412218.

[27] M. Braun, Eur. Phys. J. C16 (2000) 337; N. Armesto and M. Braun, ibid. C20 (2001) 517.

[28] A.H. Mueller, A.I. Shoshi and S.M.H. Wong, "Extension of the JIMWLK equation in the low gluon density region, arXiv:hep-ph/0501088.

[29] J.-P. Blaizot, E. Iancu and H. Weigert, Nucl. Phys. A713 (2003) 441. 
[30] E. Iancu, A. Leonidov and L. McLerran, The Colour Glass Condensate: An Introduction, hep-ph/0202270. Published in QCD Perspectives on Hot and Dense Matter, Eds. J.P. Blaizot and E. Iancu, NATO Science Series, Kluwer, 2002;

E. Iancu and R. Venugopalan, The Color Glass Condensate and High Energy Scattering in QCD, hep-ph/0303204. Published in Quark-Gluon Plasma 3, Eds. R.C. Hwa and X.N. Wang, World Scientific, 2003;

R. Venugopalan, The Color Glass Condensate: A summary of key ideas and recent developments, arXiv:hep-ph/0412396;

H. Weigert, Evolution at small $x_{\mathrm{bj}}$ : The Color Glass Condensate, arXiv:hep-ph/0501087.

[31] E. Iancu and A.H. Mueller, Nucl. Phys. A730 (2004) 460.

[32] Y. Hatta, E. Iancu, K. Itakura, L. McLerran, "Odderon in the Color Glass Condensate", arXiv:hep-ph/0501171.

[33] Yu.V. Kovchegov, Phys. Rev. D60 (1999), 034008; ibid. D61 (2000) 074018.

[34] E. Levin and M. Lublinsky, "Towards a symmetric approach to high energy evolution: generating functional with Pomeron loops", arXiv:hep-ph/0501173. 